\title{
Metal-Controlled Magnetoresistance at Room Temperature in Single-Molecule Devices
}

\author{
Albert C. Aragonès, ${ }^{\dagger, \$,}$ Daniel Aravena, ${ }^{\# ¥}$ Francisco J. Valverde-Muñoz, \\ José Antonio Real, ${ }^{\perp}$ Fausto Sanz, ${ }^{\dagger, \S}$, Ismael Díez-Pérez ${ }^{\dagger, \ldots, s^{*}}$, Eliseo Ruiz, ${ }^{\diamond, * *}$ \\ ${ }^{\dagger}$ Departament de Ciència de Materials i Química Física, Universitat de Barcelona, Martí i Franquès 1 and \\ Institut de Bioenginyeria de Catalunya (IBEC) Baldiri Reixac 15-21, Barcelona 08028 Spain \\ ${ }^{\S}$ Centro Investigación Biomédica en Red (CIBER-BBN). Campus Río Ebro-Edificio I+D, Poeta Mariano \\ Esquillor s/n, 50018 Zaragoza, Spain. \\ \# Departamento de Química de los Materiales, Facultad de Química y Biología, Universidad de Santiago de \\ Chile (USACH), Casilla 40, Correo 33, Chile \\ ${ }^{¥}$ Centro Para El Desarrollo de Nanociencias y Nanotecnología, CEDENNA, Santiago, Chile \\ ${ }^{\perp}$ Institut de Ciència Molecular (ICMol), Universitat de València, 46980 Paterna, València, Spain \\ ${ }^{\diamond}$ Departament de Química Inorgànica i Orgànica, Diagonal 645, 08028 Barcelona, Spain \\ ${ }^{\dagger}$ Institut de Química Teòrica i Computacional, Universitat de Barcelona, Diagonal 645, 08028 Barcelona, \\ Spain
}

\begin{abstract}
The appropriate choice of the transition metal complex and metal surface electronic structure opens the possibility to control the spin of the charge carriers through the resulting hybrid molecule/metal spinterface in a single-molecule electrical contact at room temperature. The single-molecule conductance of a $\mathrm{Au} /$ molecule/Ni junction can be switched by flipping the magnetization direction of the ferromagnetic electrode. The requirements of the molecule are not just the presence of unpaired electrons, the electronic configuration of the metal centre have to provide occupied or empty orbitals that strongly interact with the junction metal electrodes and that are close in energy to their Fermi levels for one of the electronic spins only. The key ingredient for the metal surface is to provide an efficient spin texture induced by the spin-orbit coupling in the topological surface states that results in an efficient spin-dependent interaction with the orbitals of the molecule. The strong magnetoresistance effect found in this kind of single-molecule wires opens a new approach for the design of room temperature nanoscale devices based on spin-polarized currents controlled at molecular level.
\end{abstract}

Keywords: Single-Molecule Junctions, Magnetoresistance, Density Functional Calculations, Spinterface, Spin Orbit Coupling 


\section{INTRODUCTION}

Many new transport properties have been recently found by combining the spin of magnetic materials with large spin-orbit coupling (SOC) effects. ${ }^{1}$ The control of the spin is one of the main challenges in the Spintronics field, and it has been already shown a large number of remarkably performing devices based mainly in multi-layered materials. ${ }^{2,3}$ Most of such devices have been built with a non-magnetic layer sandwiched between two magnetic electrodes showing, among others, magnetoresistance properties. The use of molecular entities in spintronic devices could add new dimensionalities thanks to their longer spin relaxation times, low cost and self-assembling capabilities. ${ }^{4}$ However, the use of molecules in this research field is relatively new and most of the reported experiments have been performed far from room conditions, such as ultrahigh vacuum and low temperatures. A wide fan of examples of molecular devices displaying spin-dependent functionalities have been proven at low dimensionality, ranging from the nano-scale down to the single-molecule level. For instance, the characterization of magnetoresistive molecular nanoscale devices based on terbium ${ }^{\mathrm{III}}$ complexes $^{5,6}$ resulted in a number of spin-based properties such as spin valves, ${ }^{7}$ spin transistor ${ }^{8}$ or spin resonators. ${ }^{9}$ Also, slight changes in the magnetoresistance properties have been observed in single-molecule junctions built with diamagnetic molecules connected to Ni beads. ${ }^{10,11}$ All the above examples were typically conducted at cryogenic temperatures where the intrinsic molecular magnetic properties are preserved. To the best of our knowledge, there are only two reported molecular-based devices displaying room-temperature spin-dependent transport: (i) self-assembled monolayers (SAMs) of chiral structures such as DNA deposited on gold surfaces $;{ }^{12,13}$ here, the proposed mechanism, Chiral Induced Spin Selectivity (CISS), is a combined effect of spin-orbit interaction in the chiral molecular backbone ${ }^{14,15}$ and the induced Au-S bond magnetization at the molecule--metal interface. ${ }^{16,17}$ Recently, such systems have been reviewed by Naaman and coworkers ${ }^{18,19}$ Furthermore, this effect also has been investigated in the singlemolecule breakjuntion STM technique, consisting of individual peptide molecules of well-defined chirality bridged between a magnetized STM Ni tip and an Au. ${ }^{20}$ And (ii), single-molecule 
junctions built by bridging individual small magnetic molecules such as spin-crossover $\mathrm{Fe}^{\mathrm{II}}$ complexes $\left[\mathrm{Fe}(\text { tzpy })_{2}(\mathrm{NCX})_{2}\right],(\mathrm{X}=\mathrm{S}$ or $\mathrm{Se})$ in a high-spin $\mathrm{S}=2$ configuration. $^{21}$ The observed room-temperature magnetoresistance in this case occurs when the electrons are injected from the gold (large spin-orbit effect) to a magnetic nickel electrode through the $\mathrm{Fe}^{\mathrm{II}}$ complex that acts as spin filter. ${ }^{22-27}$ The single-molecule magnetoresistance effect disappears either employing low-spin $\mathrm{S}=0 \quad \mathrm{Fe}^{\mathrm{II}}$ complexes (also by decreasing the temperature that switches the high-spin $\left[\mathrm{Fe}(\text { tzpy })_{2}(\mathrm{NCX})_{2}\right]$ complex to the non-magnetic low-spin state) or by using a $\mathrm{Cu}$ substrate (small spin-orbit) instead of $\mathrm{Au}$. In the last two examples, a fundamental difference from previous experiments is the likely requirement of a heavy diamagnetic electrode as one of the electrodes of the molecular device.

In this contribution, we design, synthetize and characterize single-molecule spin-dependent transport of a series of metal complexes $\left[\mathrm{M}(\mathrm{tzpy})_{2}(\mathrm{NCSe})_{2}\right](\mathrm{M}=\mathrm{Mn}, \mathrm{Co}$ and Ni) with two axial $\mathrm{SeCN}$ anchoring groups (see Figure 1) that were proven to be a mechanically stable metalmolecule attachment. ${ }^{21}$ The spin-dependent single-molecule transport study was conducted by trapping the metal complexes between a dielectric $\mathrm{Au}$ electrode (electron source) and a magnetically polarized Ni electrode (spin-polarized electron drain). We observed that the singlemolecule wire with the $\mathrm{S}=3 / 2 \mathrm{Co}^{\mathrm{II}}$-complex shows a conductance change of at least 100 -fold under opposite Ni magnetizations along the main junction axis (Figure 1), similar to those reported for the homologous $\mathrm{Fe}^{\mathrm{II}}$ complex. ${ }^{21}$ In contrast, the devices based on $\mathrm{Ni}^{\mathrm{II}}$ and $\mathrm{Mn}^{\mathrm{II}}$ complexes do not exhibit such magnetoresistance. Our theoretical calculations indicate that the presence of unpaired electrons in the molecule is mandatory, but that the metal electronic configuration is the key factor. The $\mathrm{t}_{2 \mathrm{~g}}{ }^{5} \mathrm{e}_{\mathrm{g}}{ }^{2}$ electronic configuration of the $\mathrm{Co}^{\mathrm{II}}$-complex provides an efficient spin-selective channel due to the presence of one occupied and one empty $\beta$ orbitals close to the Fermi level of the junction electrodes. Contrarily, for the $\mathrm{t}_{2 \mathrm{~g}}{ }^{6} \mathrm{e}_{\mathrm{g}}{ }^{2}$ electronic configuration of the $\mathrm{S}=1 \mathrm{Ni}^{\mathrm{II}}$-complex, the proximal $\mathrm{e}_{\mathrm{g}}$ orbital to the electrodes Fermi energy lacks the appropriate symmetry to mix with the 
$\pi$ orbitals of the $-\mathrm{SeCN}$ ligands that interact with the electrodes. In the $\mathrm{t}_{2 \mathrm{~g}}{ }^{3} \mathrm{e}_{\mathrm{g}}{ }^{2}$ electronic configuration of the $\mathrm{S}=5 / 2 \mathrm{Mn}^{\mathrm{II}}$-complex, the absence of the observed magnetoresistance is explained by the lack of $\mathrm{Mn}^{\mathrm{II}}$ orbitals close to the electrodes Fermi levels. The differences in the molecule/ $\mathrm{Au}$ spinterface between the two axial directions of the Ni magnetic polarization ${ }^{21}$ are indispensable to explain the observed magnetoresistance in the former (Figure 1).

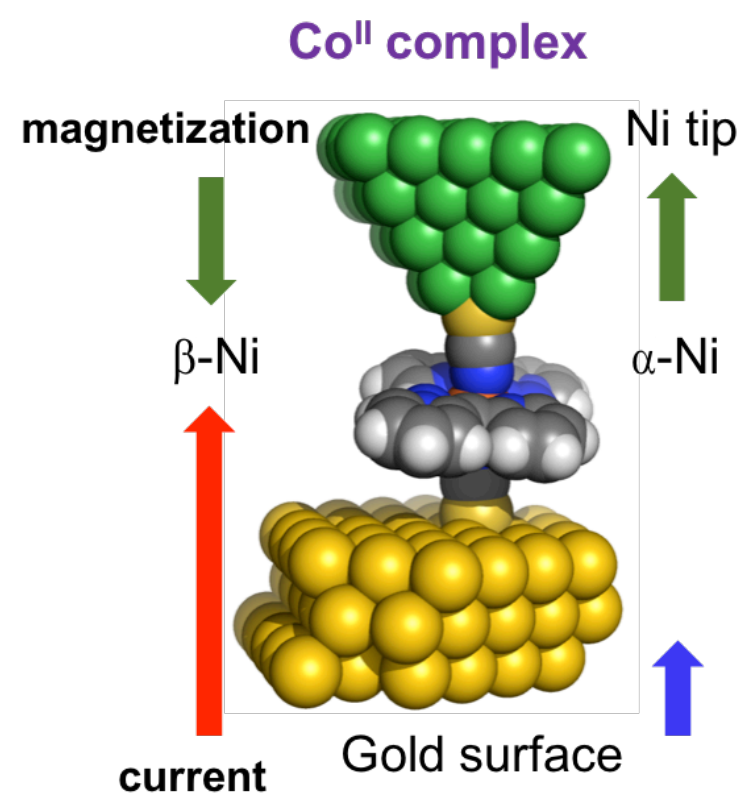

\section{$\mathrm{Nill}$ and $\mathrm{Mn}^{\|}$complexes}

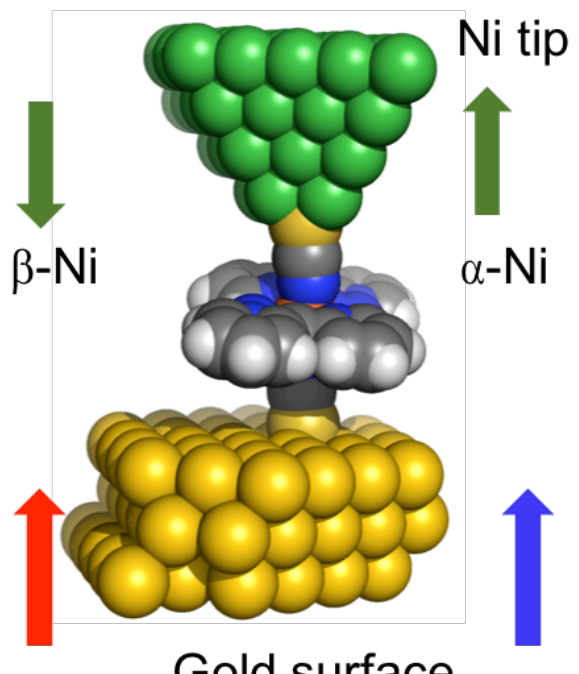

Gold surface

Figure 1. Schematic representation of the single-molecule devices studied under two opposite Ni tip magnetic polarizations (green arrows, labelled $\alpha$ and $\beta$ ). Among the paramagnetic $\left[\mathrm{M}(\mathrm{tzpy})_{2}(\mathrm{NCSe})_{2}\right]$ complexes $(\mathrm{M}=\mathrm{Mn}, \mathrm{Co}$ and $\mathrm{Ni})$, only the $\mathrm{Co}^{\mathrm{II}}$ complex shows high tunneling magnetoresistance (red and blue arrows indicate currents for the two opposite magnetizations of the Ni tip), while transport in the $\mathrm{Mn}^{\text {II }}$ and $\mathrm{Ni}^{\mathrm{II}}$ complexes remains invariable versus the $\mathrm{Ni}$ magnetization directions.

\section{RESULTS AND DISCUSSION}

Room-temperature single-molecule transport of the $\left[\mathrm{M}(\mathrm{tzpy})_{2}(\mathrm{NCSe})_{2}\right]$ complexes $(\mathrm{M}=\mathrm{Mn}$,

Co and Ni). Single-molecule conductance experiments were performed at room temperature using

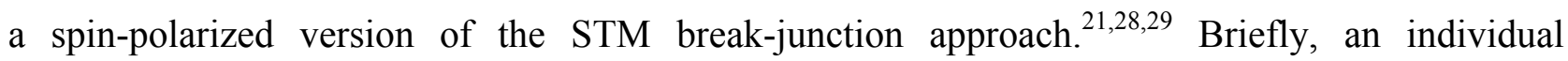
$\left[\mathrm{M}(\text { tzpy })_{2}(\mathrm{NCSe})_{2}\right]$ complex $(\mathrm{M}=\mathrm{Mn}, \mathrm{Co}$ and $\mathrm{Ni})$ was trapped between an $\mathrm{Au}$ and a ferromagnetic $\mathrm{Ni}$ electrode under opposite magnetic polarization directions along the axial molecular junction (Figure 1). For this purpose, a freshly mechanically cut Ni tip was magnetically polarized ex situ by placing it in close proximity to a $1 \mathrm{~T} \mathrm{NdFeB}$ magnet for a period of 2 hours, and then placed into 
the STM tip holder. To avoid Ni oxidation during the magnetization stage, the Ni tip was kept under anaerobic conditions (see detailed Ni tip preparation and characterization elsewhere ${ }^{21}$ ). The magnitude and direction of the magnetic polarization of the Ni tip were characterized before and after the STM break-junction experiment using SQUID magnetometry to check that they persisted over the entire timeframe of the experiments. ${ }^{21}$ The magnetized STM Ni probe was then driven toward the surface and pulled back again in successive cycles, using a 2-point feedback loop on the tunneling current flowing between the two electrodes under a constant bias voltage. Representative current traces during the pulling stage are shown in the insets of Figures 2a-c for the three $\left[\mathrm{M}(\text { tzpy })_{2}(\mathrm{NCSe})_{2}\right]$ complexes $(\mathrm{M}=\mathrm{Mn}, \mathrm{Co}$ and $\mathrm{Ni})$, respectively. When a molecule bridges between the two electrodes, a plateau appears in the current trace during the pulling stage at a specific molecular conductance. ${ }^{28,30}$ The absence of other exposed interacting groups in these compounds as well as the single plateau features in the decay curves suggests the axial $\mathrm{SeCN}$ ligand as the only anchoring points. ${ }^{21}$ Hundreds of these individual traces are then accumulated into the same conductance histogram where the average single-molecule conductance value is extracted from the Gaussian fitting of the peaks (Figures 2a-c). The $\alpha$ and $\beta$ labels correspond to the magnetic moment of the Ni electrode, pointing upward and downward respectively, along the main molecular junction axis (see Figure 1).

The analysis of the histograms in Figure 2 reveals two markedly different charge transport behaviors: (i) the single-molecule junctions built with the $\mathrm{Mn}^{\mathrm{II}}$ and $\mathrm{Ni}^{\mathrm{II}}$ complexes display similar characteristic conductance values for both $\alpha$-up and $\beta$-down directions of the Ni magnetization (see Figures $2 \mathrm{a}$ and c) and, consequently, no magnetoresistance. The $\left[\mathrm{Mn}(\operatorname{tzpy})_{2}(\mathrm{NCSe})_{2}\right]$ displays a conductance around $3.8 \cdot 10^{-4} \mathrm{G}_{0}$ (being $\left.\mathrm{G}_{0}=77.5 \mu \mathrm{S}\right), \sim 5$-fold higher that of the $\left[\mathrm{Ni}(\text { tzpy })_{2}(\mathrm{NCSe})_{2}\right.$, $8.2 \cdot 10^{-5} \mathrm{G}_{0}$. (ii) Contrarily, the single-molecule junctions built with the $\left[\mathrm{Co}(\mathrm{tzpy})_{2}(\mathrm{NCSe})_{2}\right]$ complex displays a measurable conductance value for the $\beta$-down polarized $\mathrm{Ni}$ electrode only (Figure 2b). This measured magnetoresistance for the $\mathrm{Co}^{\mathrm{II}}$ compound is comparable to that 
previously reported for a homologous $\mathrm{Fe}^{\mathrm{II}}$ complex while the obtained conductance value, $1.39 \cdot 10^{-}$

${ }^{3} \mathrm{G}_{0}$, is $\sim 3$-fold larger than the previously reported for the equivalent $\left[\mathrm{Fe}(\mathrm{tzpy})_{2}(\mathrm{NCSe})_{2}\right]$ ) complex, $4.4 \cdot 10^{-4} \mathrm{G}_{0}$ for the same $\beta$-down $\mathrm{Ni}$ polarization direction. ${ }^{21}$ No current plateaus were then appreciable in the individual pulling traces for the $\left[\mathrm{Co}(\text { tzpy })_{2}(\mathrm{NCSe})_{2}\right]$ complex in the singlemolecule experiments with $\alpha$-up polarized $\mathrm{Ni}$ electrodes (gray traces in the Figure $2 \mathrm{~b}$ inset). The single-molecule conductance is in this case below the detection limit of our current amplifiers $(<$ $10^{-6} \mathrm{G}_{0}$, see gray histogram in Figure $2 \mathrm{~b}$ ).
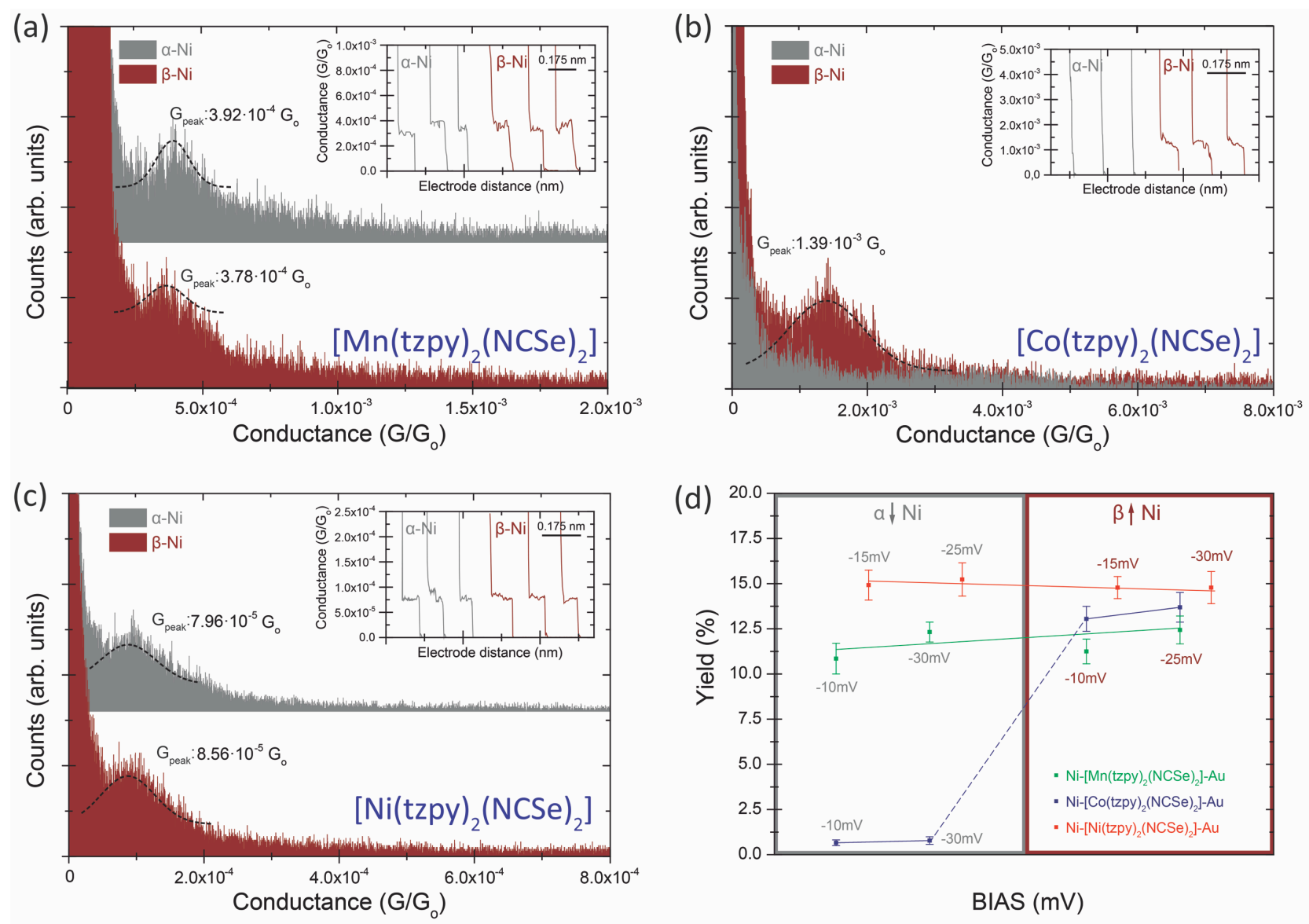

Figure 2. Single-molecule conductance histogram for the metal complexes: (a) $\mathrm{S}=5 / 2$ [Mn(tzpy $)_{2}(\mathrm{NCSe})_{2}$, (b) $\mathrm{S}=3 / 2\left[\mathrm{Co}(\text { tzpy })_{2}(\mathrm{NCSe})_{2}\right]$ and (c) $\mathrm{S}=1\left[\mathrm{Ni}(\text { tzpy })_{2}(\mathrm{NCSe})_{2}\right]$ bridging between $\mathrm{Au}$ and both $\alpha$-up polarized (gray) and $\beta$-down (maroon) magnetically polarized Ni electrodes. The two histograms in (a) and (c) have been vertically offset for clarity. All conductance values have been extracted from Gaussian fits of the peaks. Insets show representative individual current versus pulling traces for the $\alpha$-up polarized (left) and the $\beta$-down polarized (right) $\mathrm{Ni}$ electrodes used to build the conductance histograms. The applied bias was set to $-15 \mathrm{mV}$ in (c) and $-25 \mathrm{mV}$ in (a) and (b) as indicated in the figure. (d) Single-molecule junction yield (\%) for the three compounds under the two Ni magnetic polarizations. Different points correspond to different employed bias voltages and the error bars denote the standard deviation from the average conductance results of different experiments. 
Figure $2 \mathrm{~d}$ summarizes the experimental yield of the single-molecule transport experiments for the three studied compounds under the two Ni magnetic polarizations. As previously described, ${ }^{21}$ the single-molecule yield is expressed as the percentage of individual pulling traces showing plateau features. Typically, single-molecule experimental values of $>10 \%$ are routinely obtained experimentally ${ }^{31,32}$ and allow the single-molecule conductance characterization. All three systems show yields above $10 \%$ for the formation of single-molecule junctions between the two metal electrodes for a $\beta$-down Ni polarization direction. When the Ni polarization is inverted, the yield drops down to values below $1 \%$ for the $\left[\mathrm{Co}(\mathrm{tzpy})_{2}(\mathrm{NCSe})_{2}\right]$ compound, whereas the yield remains constant for the other two.

Effect of the metal substrate on the single-molecule magnetoresistance. In order to highlight the role of the metal substrate on the observed magnetoresistance for the single-molecule contact formed with the $\left[\mathrm{Co}(\mathrm{tzpy})_{2}(\mathrm{NCSe})_{2}\right]$ compound, we have performed single-molecule charge transport measurements on $\mathrm{M} /\left[\mathrm{Co}(\text { tzpy })_{2}(\mathrm{NCSe})_{2}\right] / \mathrm{Ni}$ junctions $(\mathrm{M}=\mathrm{Au}, \mathrm{Cu}, \mathrm{Pt})$ under opposite magnetization directions of the Ni tips along the main molecular junction axis. Due to the poor mechanical ductility of the Ni-Pt junction, the length of the plateaus in the break-junction pulling traces resulting from the single-molecule bridge formation was almost unappreciable, ${ }^{33}$ fact that forced us to exploit a previously presented blinking approach to build and characterize the singlemolecule conductance (see a detailed description elsewhere ${ }^{34,35}$ ). Briefly, in the blinking approach, the Ni STM electrode is held at a nanometer distance from the metal surface. When a molecule spans the gap, a telegraphic signal is recorded in the current transient (see Figure 3 left panels) whose amplitude equals the conductance of the molecular junction. The existence of a molecular bridge is ascertained by performing pulling stages during the $\mathrm{ON}$ telegraphic signal to promote the molecular breakdown (see Supporting Information section 5). Up to a hundred of the individual 
blinking traces are accumulated in a 2D blinking map without any selection by setting all of them to a common time origin and background tunneling current subtraction.
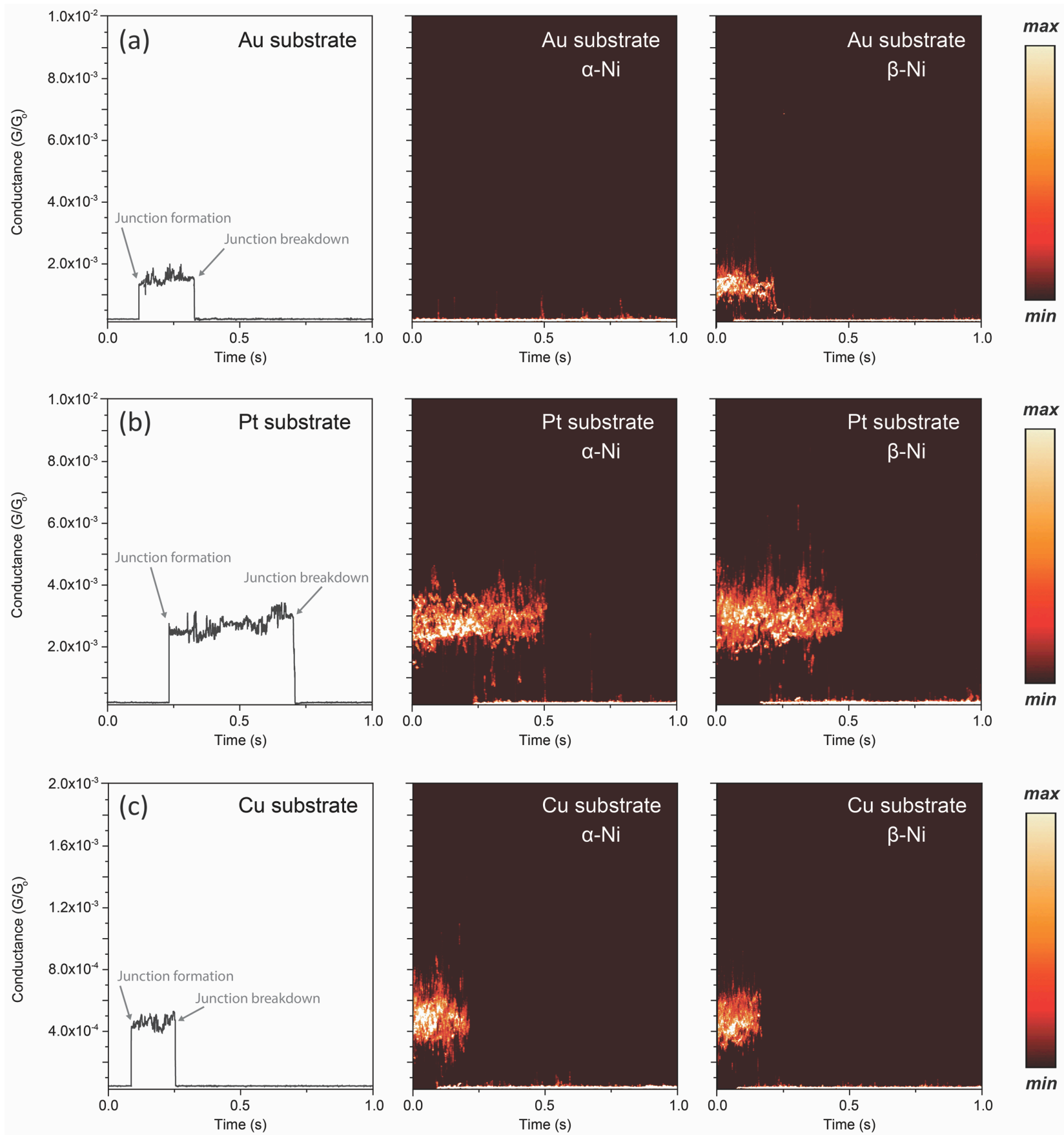

Figure 3. Single-molecule charge transport of the $\left[\mathrm{Co}(\mathrm{tzpy})_{2}(\mathrm{NCSe})_{2}\right]$ compound on different metal surfaces. The horizontally expanded panels show, from left to right, a representative blinking event of the single-molecule bridge formation, and the two 2D blinking maps showing the average of several tens of individual blinking traces for the two opposite directions of the $\mathrm{Ni}$ electrode magnetization $\alpha$-/ $\beta$ respectively (see Figure 1) on (a) Au, (b) Pt and (c) Cu surfaces. The 2D-maps were normalized to a color scale versus the total number of counts, representing 100 counts the maximum and 0 counts the minimum. The applied bias was set to $-10 \mathrm{mV}$. 
Figure 3 shows a comparison of the three $2 \mathrm{D}$ blinking maps recorded for the $\left[\mathrm{Co}(\mathrm{tzpy})_{2}(\mathrm{NCSe})_{2}\right]$ single-molecule junctions formed between the magnetically polarized Ni STM electrode and an $\mathrm{Au}, \mathrm{Pt}$ and $\mathrm{Cu}$ surfaces, respectively. The central and right panels correspond to the 2D blinking maps with the Ni electrode magnetically polarized upward and downward respectively. The results obtained using the Au substrate (Figure 3a) are essentially the same shown in Figure 2b employing the break-junction approach, i.e. the level of conductance and the magnetoresistance effect displaying suppression of the single-molecule conductance at the $\alpha$-up Ni magnetization direction are equally equally observed. Contrarily, the molecular junctions built on either $\mathrm{Pt}$ or $\mathrm{Cu}$ surfaces are insensitive to the magnetic polarization direction of the top Ni electrode, showing essentially no magnetoresistance of the single-molecule wire conductance for the $\left[\mathrm{Co}(\mathrm{tzpy})_{2}(\mathrm{NCSe})_{2}\right]$ complex (Figures $3 \mathrm{~b}$ and $3 \mathrm{c}$ respectively). The suppression of magnetoresistance observed in the singlemolecule wire built on $\mathrm{Cu}$ was also confirmed by characterizing the single-molecule transport of $\left[\mathrm{Co}(\text { tzpy })_{2}(\mathrm{NCSe})_{2}\right]$ using the previous dynamic STM break-junction approach (see Supporting Information section 3). These results evidence the importance that molecule/metal interfacial effects have on the final observe device magnetoresistance as it will be discussed in the next section.

Spin-dependent density of states and transmission calculation. To rationalize the origin of the observed differences in magnetoresistance in the studied single-molecule junctions, we first performed spin-polarized DFT-NEGF calculations. ${ }^{36-38}$ The structural model for the calculations consisted in two true semi-infinite Au surface electrodes with the molecule attached to both $\mathrm{Au}(111)$ surface by the $\mathrm{Se}$ atoms in a three-fold hollow configuration. The calculations were carried out using two equivalent gold surfaces in order to simplify the system and considering that the spin-filter effect arises from the mixing of the gold surface levels with the spin-polarized molecular orbitals. ${ }^{21}$ The nickel tip is not included in the calculation since it just controls the final device conductance because the transport of minority spin carriers is more efficient in the magnetically polarized Ni electrode. ${ }^{39}$ Furthermore, the magnetic effect of the nickel tip on the 
molecule is included using a spin-polarized single determinant to describe the electronic structure of the molecule.
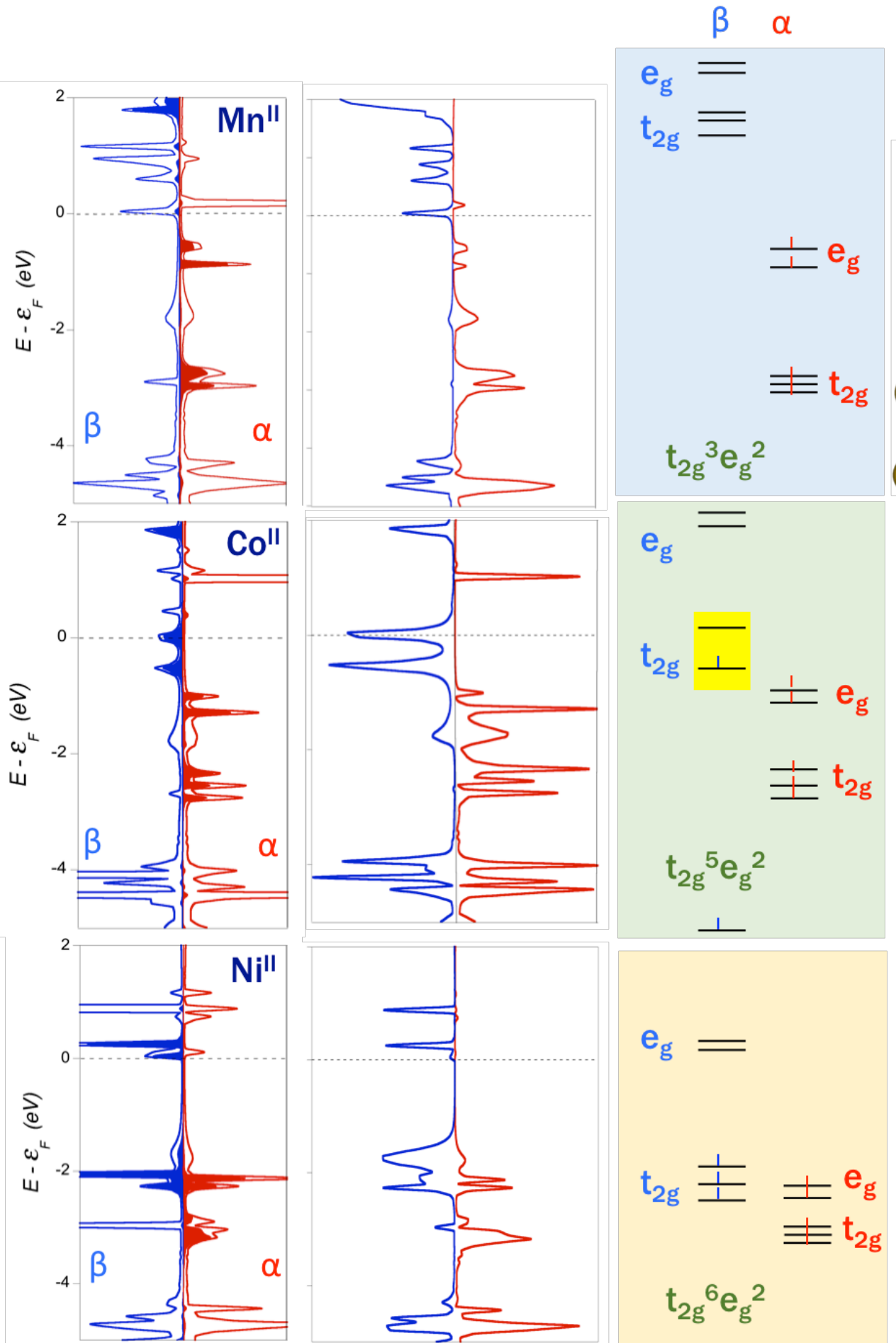

Figure 4. DFT calculated spin-resolved projected density of states (left) of the $\mathrm{Mn}^{\mathrm{II}}, \mathrm{Co}^{\mathrm{II}}$ and $\mathrm{Ni}^{\mathrm{II}}$ complexes (filled curve corresponds to the transition metal levels and metal orbitals and their occupation are represented in the right side) and transmission spectra (right, between 0 and 2 for each spin) for a $2.5 \AA$ constant gold-ligand bond distance. Red and blue colors correspond to the alpha and beta spin contributions, respectively. Transition metal $d$ orbitals out of the energy range have been just placed without considering their orbital energy values. The highlighted $\mathrm{t}_{2 \mathrm{~g}}$ unpaired beta orbitals in the $\mathrm{Co}^{\mathrm{II}}$ complex correspond to the main spin-dependent electron channels. 
The calculated density of states (DOS) and transmission curves for the three complexes $(\mathrm{M}=\mathrm{Mn}$, Co and Ni) adsorbed between two Au(111) surfaces are plotted in Figure 4. The main observed differences between the three studied cases can be summarized as follow for each metal system:

(i) The Mn ${ }^{\mathrm{II}}$ system has $\mathrm{at}_{2 \mathrm{~g}}{ }^{3} \mathrm{eg}_{\mathrm{g}}{ }^{2}$ electronic configuration, where either the highest occupied alpha $\mathrm{e}_{\mathrm{g}}$ orbital and the lowest unoccupied beta $\mathrm{t}_{2 \mathrm{~g}}$ orbitals are far from the Au Fermi level (around 0.5 $\mathrm{eV}$ below and $1.8 \mathrm{eV}$ above in the DOS, Figure 4 left and right panels, respectively). Hence, despite that it is the molecule with largest total spin $S=5 / 2$, the single-molecule device is unlikely to show magnetoresistance effects at low applied voltage biases as experimentally observed. Despite the lack of $3 \mathrm{~d}$ orbitals close to the Fermi energy, there is a non-negligible contribution to $\mathrm{T}(\mathrm{E})$ starting from the Fermi level (Figure 4, central panel) that corresponds to narrow levels with a poor interaction with the electrodes. Furthermore, the states contributing to the $T(E)$ in this range are highly delocalized unoccupied orbitals, which appear too close to the Fermi level due to the typical gap underestimation of the PBE functional. We expect these states to be further away from the Fermi level, having a much lower contribution to the electronic transmission. To check this assumption, we performed a model calculation of the $\mathrm{Mn}^{\mathrm{II}}$ system coupled to two small gold clusters and obtained the transmission function for zero bias. We employed the B3LYP functional to incorporate an improved description of the HOMO-LUMO gap (see Computational Details section for further reference). We observe a notorious increase of the energy gap, where the main contributions to the electronic transport come from d-orbital levels that are far from the Fermi level ( 1.5-2.0 eV apart, see Figure 5). This is in line with the relatively low single-molecule conductance of this system $\left(\sim 3.8 \cdot 10^{-4} \mathrm{G}_{0}\right.$, see Figure $\left.2 \mathrm{a}\right)$.

(ii) The Co ${ }^{\mathrm{II}}$ case displays two $t_{2 \mathrm{~g}}$ beta orbitals (highlighted in yellow in Figure 4 right) that are closer to the Au electrode Fermi level in comparison with the alpha channel orbitals that display the highest occupied orbitals appearing $\sim 1.0 \mathrm{eV}$ apart. The observed broadening of these two $\mathrm{t}_{2 \mathrm{~g}}$ 
beta orbitals evidences its effective hybridization to the metal orbitals, which also results in a higher measured conductance of the single-molecule junction for this molecular system through the beta orbital channel $\left(\sim 1.4 \cdot 10^{-3} \mathrm{G}_{0}\right.$, see Figure $\left.2 \mathrm{~b}\right)$. The analysis of such two orbitals shows correspondence to the $\pi$ antibonding $d_{x z}$ and $d_{y z} t_{2 g}$ orbitals with strong interaction with the gold electrode through the $\pi$ system of the axial ligands, while the third $t_{2 g}$ orbital (non-bonding $d_{x y}$ ) is located below $-5 \mathrm{eV}$. B3LYP results for the corresponding model system support this picture, with relatively broad transmission peaks that are 1.0 to $1.5 \mathrm{eV}$ apart from the Fermi level (see Figure 5) suggesting that the transport proceeds through the $\beta$ LUMO. As a result, $\beta$ spin-polarized orbital channels are responsible for the pronounced spin filtering of the current transmitted through the $\mathrm{Co}^{\mathrm{II}}$-complex single-molecule junction. We have calculated the energy involved in the flipping of the molecular spin with the complex adsorbed on a hollow site of a spin-polarized Ni(111) surface. The value is $90 \mathrm{~cm}^{-1}$ favorable for the parallel spin alignment using the PBE functional (see Computational detail section). Considering the magnetically polarized Ni tip induces the same spin alignment at the molecule through magnetic exchange interactions, ${ }^{40}$ the observed single-molecule magnetoresistance is explained by an asymmetric hybridization of the molecular orbitals to the magnetically polarized molecule/Au interface (spinterface). ${ }^{41,42}$ This effect is similar to that presented in the previously reported $\mathrm{Fe}^{\mathrm{II}}$ systems, which also displays DOS and transmission curves with two of the beta $t_{2 g}$ orbitals close to the Fermi level. ${ }^{21,22}$ The role of the spinterface effect in the appearance of magnetoresistance in the single-molecule junction built with the $\mathrm{Co}^{\mathrm{II}}$ system is corroborated in the experimental results shown in Figure 3, where the interfacial magnetic polarization is suppressed by using other metal surfaces (e.g. $\mathrm{Cu}, \mathrm{Pt}$ magnetoresistance for the $\mathrm{Co}^{\mathrm{II}}$ system vanishes in Figures 3b-c) with less tendency to be spin-polarized than Au. Hence, the gold surface is a key ingredient to produce the magnetoresistance behavior due to the existence of spin unbalanced topological surface states detected by angle-resolved photoemission spectroscopy (ARPES), ${ }^{43}$ wherein the spin-orbit effect results in spin texture on this surface. Although gold, copper and platinum exhibit the same qualitative structure with respect to their sp- 
band crossing, ${ }^{43}$ we observe that magnetoresistance effects are only observed when the Co ${ }^{\mathrm{II}}$ complex is deposited on gold. In the case of $\mathrm{Cu}$, this behavior is related to the low spin-orbit coupling constant due to its lower atomic weight. In the case of Pt, despite a similar structure of gold, it has one valence electron less that yields a different mixing with the $\mathrm{SeCN}^{-}$ligands in comparison to $\mathrm{Au}$. We will come back to this point in the next section.

(iii) The $\mathrm{Ni}^{\mathrm{II}}$ system is similar to the $\mathrm{Mn}^{\mathrm{II}}$ case, as it has delocalized orbitals close to the Fermi level for both beta and alpha electrons, with a higher beta contribution (Figure 4, right panel). In this case, the beta empty $e_{g}$ orbitals also appear relatively close to the Fermi level. The corresponding transmission peaks are narrow manifesting the very weak mixing of the frontier $\sigma \mathrm{e}_{\mathrm{g}}$ orbitals of the $\mathrm{Ni}^{\mathrm{II}}$ complex to the $\pi$ nature of axial $\mathrm{SeCN}$ orbitals that interacts with the gold surface levels. This poor hybridization also explains the low conductance measured in this case $\left(\sim 8.6 \cdot 10^{-5} \mathrm{G}_{0}\right.$, Figure $\left.2 \mathrm{c}\right)$. The B3LYP calculation effectively corrects for the position of the delocalized empty orbitals, which appear now far from the Fermi level (see Figure 5). Furthermore, the transmission peaks corresponding to $e_{g}$ orbitals are extremely narrow, confirming the low mixing of these orbitals and the low experimental conductivity of the $\mathrm{Ni}^{\mathrm{II}}$ derivative. Figure 5 shows satisfactorily the relation between the measured conductance and the energy profile of the transmission function. The most conductive ion is $\mathrm{Co}^{\mathrm{II}}$, in agreement with the experimental data, due to their highly coupled empty $t_{2 g}$ levels that appear relatively close to the Fermi level (indicated with a blue arrow in Fig. 5) The less conducting cases are observed for the $\mathrm{Mn}^{\mathrm{II}}$ and $\mathrm{Ni}^{\mathrm{II}}$ complexes that only present poorly mixing of the $e_{\mathrm{g}}$ type orbitals near the Fermi level. If we include the Fe ${ }^{\mathrm{II}}$ derivative to this analysis, we observe that it presents lower conductivity than $\mathrm{Co}^{\mathrm{II}}$, but higher than $\mathrm{Mn}^{\mathrm{II}}$ and $\mathrm{Ni}^{\mathrm{II}}$. Similar to the case of $\mathrm{Co}^{\mathrm{II}}$, this system presents significantly broadened beta $\mathrm{t}_{2 \mathrm{~g}}$ orbitals that appear further away from the Fermi level in comparison to $\mathrm{Co}^{\mathrm{II}}$ derivative, explaining its lower conductivity. ${ }^{21}$ On the other hand, the conductivity associated with $\mathrm{t}_{2 \mathrm{~g}}$ orbitals in $\mathrm{Fe}^{\mathrm{II}}$ (the last occupied $\mathrm{t}_{2 \mathrm{~g}}$ orbital of $\mathrm{Co}^{\mathrm{II}}$ close to the Fermi level is empty for $\mathrm{Fe}^{\mathrm{II}}$ and it is the main 
responsible of transport for such complex) should be higher than the contributions from $\mathrm{e}_{\mathrm{g}}$ orbitals, which are dominant for $\mathrm{Mn}^{\mathrm{II}}$ and $\mathrm{Ni}^{\mathrm{II}}$ systems. The observed lack of magnetoresistance for the $\mathrm{Mn}^{\mathrm{II}}$ and $\mathrm{Ni}^{\mathrm{II}}$ single-molecule junctions is then explained by the non-effective spin filter behavior of the molecule due to the poor differences in the transmission efficiency between the alpha and beta channels.
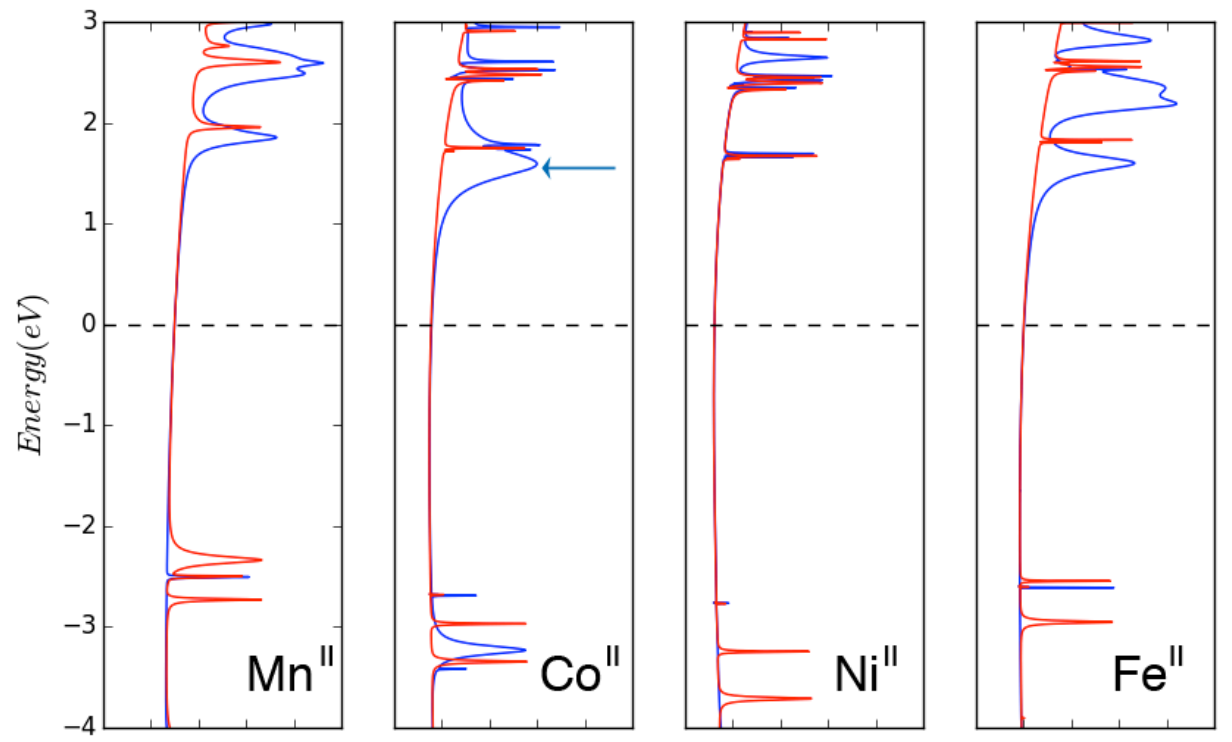

Figure 5. DFT transmission (between 0 and 2.5 for each spin) calculated with the hybrid B3LYP functional for the single $\left[\mathrm{M}(\text { tzpy })_{2}(\mathrm{NCSe})_{2}\right]$ complexes $(\mathrm{M}=\mathrm{Co}, \mathrm{Mn}, \mathrm{Ni}$ and $\mathrm{Fe})$ sandwiched between two (red and blue are alpha and beta contributions, respectively). The $\mathrm{Fe}^{\mathrm{II}}$ system is included for comparison (see text) and was investigated with periodic models elsewhere. ${ }^{21}$ The Fermi level was chosen to be $-5 \mathrm{eV}$ and set to 0 $\mathrm{eV}$ in the plot (marked with a dashed line). The blue arrow indicates in the $\mathrm{Co}^{\mathrm{II}}$ case the crucial $\mathrm{t} 2 \mathrm{~g}$ orbital for the magnetoresistance effect.

An important message is deduced from the above discussion; an appropriate electronic structure of the magnetic molecule is necessary to develop magnetoresistance in a single-molecule device, similarly to what is required to show large magnetic anisotropy effects, i.e. low-lying excitations and differentiated alpha-beta electronic configurations. ${ }^{44,45}$ The existence of low-lying excitations warranties the presence of occupied and empty orbitals in a narrow energy range that allows available channels for efficient transport if they lie close to the Fermi level of the electrodes. For instance, the $\left[\mathrm{Co}(\text { tzpy })_{2}(\mathrm{NCSe})_{2}\right]$ complex has mononuclear single-molecule magnet behavior (see Supplementary section 4.3) with a calculated axial zero field-splitting D value at the NEVPT2 level 
of $+16.7 \mathrm{~cm}^{-1}$, achieving easy-plane anisotropy as expected for a pseudo-octahedral $\mathrm{Co}^{\mathrm{II}}$ complex. $^{44}$ Thus, single-molecule magnet complexes are in principle good candidates to show appealing transport properties, such as magnetoresistance effects in single-molecule devices.

Spin texture of the gold surface. After establishing the relation between the electronic configuration of the molecule and the development of magnetoresistance in our devices, it is necessary to investigate why spin-dependent transport develops on the Au surface only, while platinum and copper junctions do not present such behavior. To this aim, we carried out an analysis of the electronic structure of one slab of the three materials to analyze their differences and, consequently, to explain the singular properties of the gold junctions. The goal will be to check if there are surface levels that can efficiently interact with the $\mathrm{t}_{2 \mathrm{~g}}$ molecular orbitals of the Co ${ }^{\mathrm{II}}$ (or Fe ${ }^{\mathrm{II}}$ complexes) through the $\pi$ orbitals of SeCN ligand in such way that the different alpha and beta occupancy in these two metal complexes could induce a different magnetic polarization of the metal surface.

We highlight the main features of the $\mathrm{Au}(111)$ DFT band structure (Figure 6, PBE functional including spin-orbit effects, see Computational details section) related to the problem of molecule surface mixing: (i) the hybridization of the $6 \mathrm{~s}$ and $6 \mathrm{p}_{z}$ levels (red and green lines respectively in Figure 6a, L-band) shows the characteristic Rashba spin-dependent splitting due the SOC of the surface state of gold (see point A in Figure 6a), (ii) a sp-band inversion at an energy larger than the Fermi level (point B) is observed in the $\Gamma \rightarrow \mathrm{M}$ path and (iii) a second band crossing involving $\mathrm{p}$ and $\mathrm{d}$ orbitals appears close to the Fermi level at the $\mathrm{M}$ point ( $\mathrm{C}$ in Figure 6a). The k-dependent band diagram (Figures $6 \mathrm{~b}$ and $6 \mathrm{c}$ ) also shows the L-band splitting near $\Gamma$ and, despite strong kdependent spin polarization of the $\mathrm{d}$ - and p-type orbitals (blue and green lines, respectively in Figure 6a under $-2 \mathrm{eV}$ ), there is a null net spin contribution along the whole Brillouin zone. 
Furthermore, point $\mathrm{C}$ presents a complex structure at the merging point of several bands with different spin polarization.
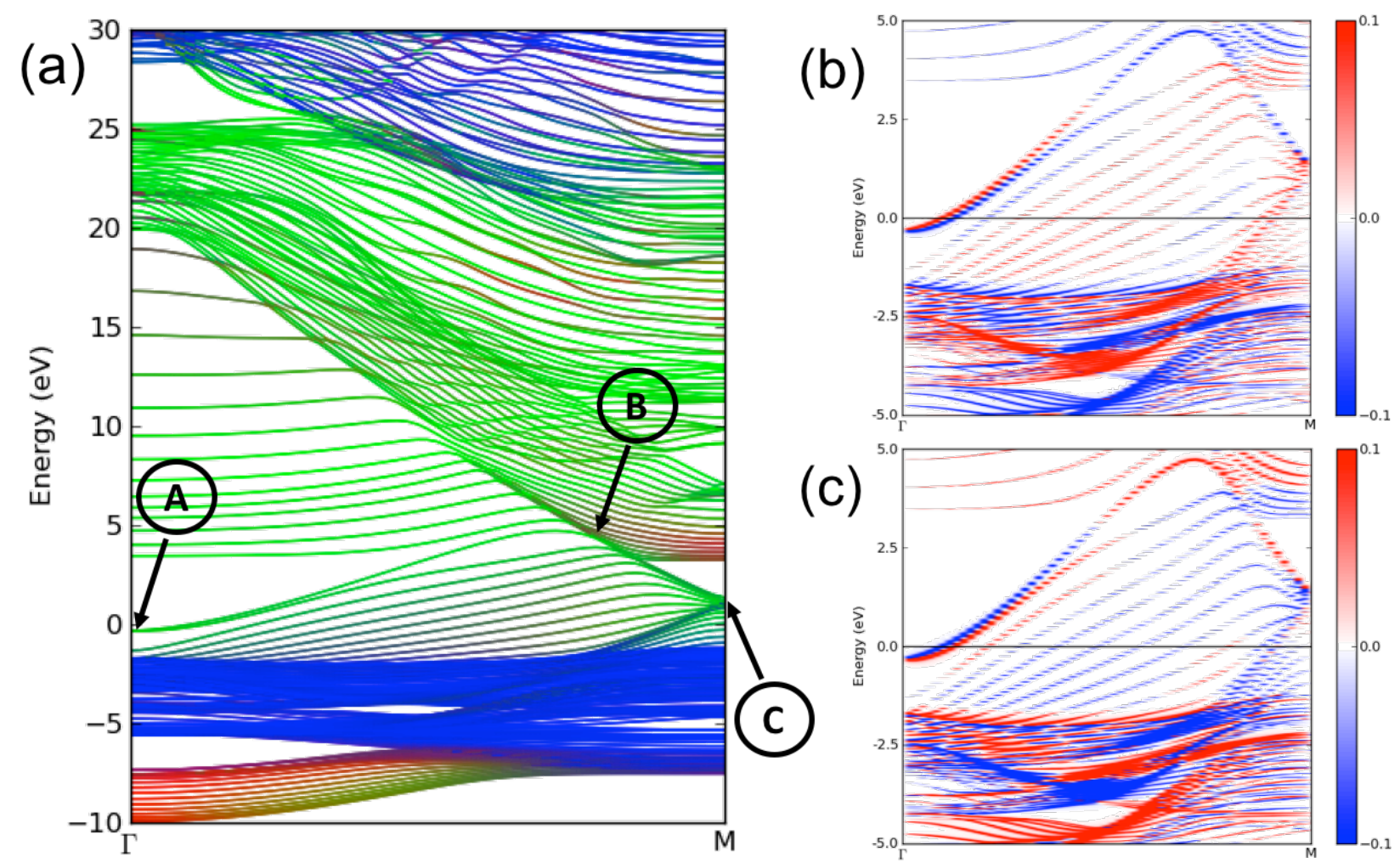

Figure 6. (a) Calculated band structure of $\mathrm{Au}(111)$ surface along the $\Gamma \rightarrow M(\pi / \mathrm{a}, 0,0)$ path. Bands were obtained from an 18-atom slab calculation of a $1 \times 1$ surface. The color of the bands represents the contributions from $\mathrm{s}, \mathrm{p}$ and $\mathrm{d}$ orbitals, coded in a RGB scheme. In this way, pure red, green and blue represent bands dominated by $\mathrm{s}, \mathrm{p}$ and $\mathrm{d}$ contributions, respectively. Intermediate colors are weighted by each contribution. (b and c) Spin polarization (red and blue colors for the two spin orientations) along the $\mathrm{x}$ (b) and $\mathrm{y}-$ (c) directions projected on one surface gold atom. There is no spin polarization along the $\mathrm{z}$ axis.

Considering that the orbitals responsible for the interaction between molecule and metal surface are the $\pi$ orbitals of the axial SeCN ligand, we must search for bands that can match this symmetry. We then performed spin polarized calculations (without SOC coupling) of a $2 \times 2 \mathrm{Au}(111)$ supercell (see details of the choice of the unit cell in Supporting Information section 7). Under this perspective, the L-band at $\Gamma$ is not adequate for mixing, as it is composed by the hybridization of the $6 \mathrm{~s}$ and $6 \mathrm{p}_{\mathrm{z}}$ gold orbitals (see Figure S12), and will form a $\sigma$-bond with respect to the molecule. The L-band at M point $(\pi / \mathrm{a}, 0,0)$ would offer a suitable orbital for mixing, as wavefunctions in neighbor atoms will present alternating signs of their wave function (a more detailed discussion of 
this surface orbitals is included in section S7). The participation of such surface orbitals is corroborated with the analysis of the main transmission eigenfunctions obtained with the ATK $\operatorname{code}^{36}$ for the two transmission peaks corresponding to the two $t_{2 g}$ orbitals $\left(d_{x z}\right.$ and $\left.d_{y z}\right)$ of the $\mathrm{Co}^{\text {II }}$ complex close to the Fermi level (see Figure 4). In the case of the $\mathrm{Co}^{\mathrm{II}}$ complex, the transport mechanism is mainly due to the transmission peak corresponding to the transmission eigenstate depicted in the right panel of Figure 7 (associated to the empty $\mathrm{t}_{2 \mathrm{~g}}$ orbital), while for $\mathrm{Fe}^{\mathrm{II}}$, with one beta electron less, is that of the left panel of Figure 7. Thus, a similar magnetoresistance behavior should be expected for these two complexes while with the other studied transition metal atoms, the complexes do not have the appropriate electronic structure, only $\mathrm{d}^{1}$ and $\mathrm{d}^{2}$ complexes would be equivalent to the $\mathrm{Fe}^{\mathrm{II}}$ and $\mathrm{Co}^{\mathrm{II}}$ cases but with opposite spin carriers.
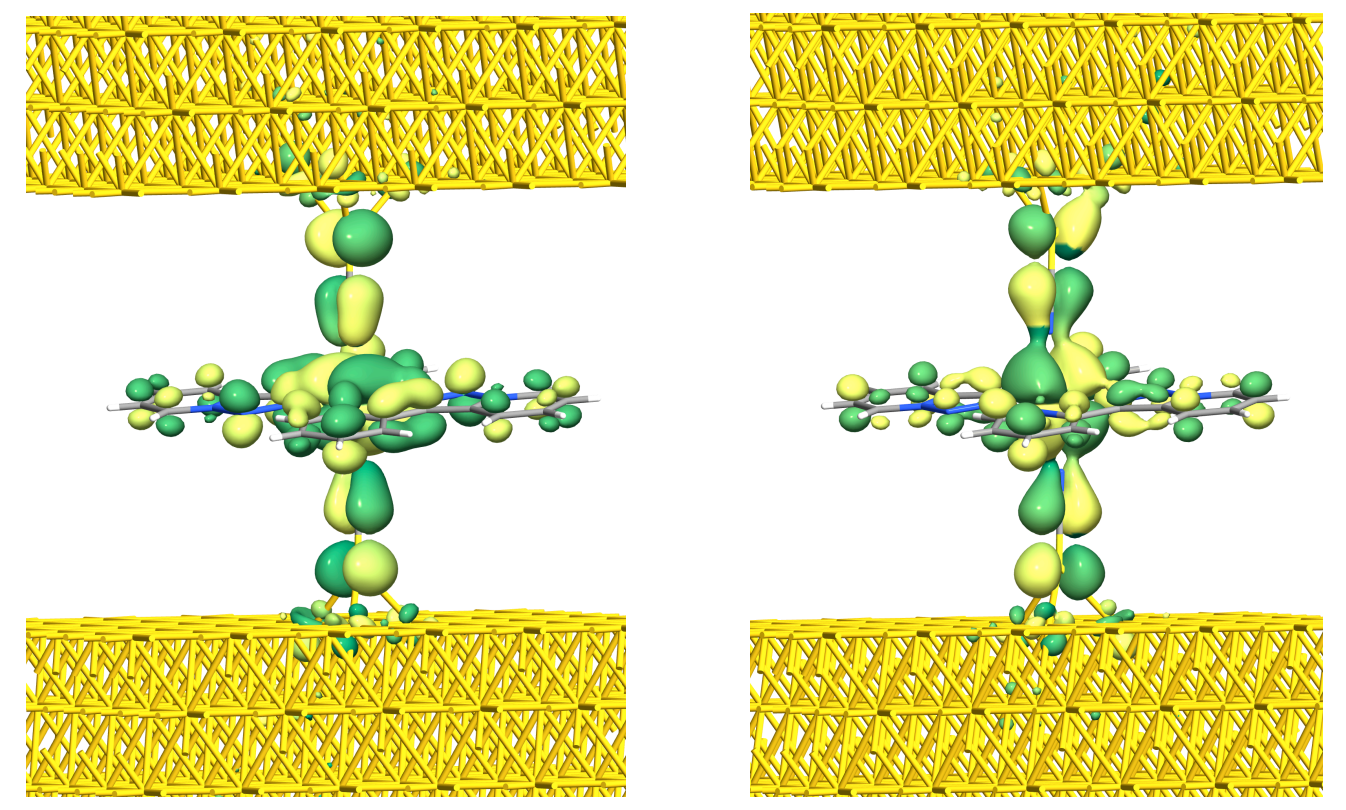

Figure 7. Main transmission eigenstates corresponding to the beta transmission peaks at -0.52 and $+0.04 \mathrm{eV}$ for the $\mathrm{Co}^{\mathrm{II}}$ complex (occupied and empty $\mathrm{t}_{2 \mathrm{~g}}$ orbitals, see Figure 4). The transmission eigenstates are obtained by diagonalizing the transmission matrix and the corresponding eigenvalues indicate the importance of each eigenstate in the transport. As it is a complex wavefunction, the color map indicating the phase of the function is represented from 0 to $2 \pi$ by dark green to yellow colors. The isovalue employed for the isosurface is 0.2 .

Analogous calculations to analyze the surface states were performed for $\operatorname{Pt}(111)$ and $\mathrm{Cu}(111)$ surfaces were also performed (see Figure 8). In the case of $\mathrm{Cu}$, we observe a band structure resembling the gold case (see Figure 8), with a surface band related to valence $4 s^{1}$ electron and sp- 
band inversion in the $\Gamma \rightarrow \mathrm{M}(\pi / \mathrm{a}, 0,0)$ path. As in the Au case, the d-based orbitals form a dense block of states that remain occupied along the Brillouin zone. This analogy is expected, as copper and gold belong to the same group of the periodic table and present the same valence configuration. We associate the lack of magnetoresistance effect on $\mathrm{Cu}$ surfaces to the low atomic number of copper, which prevents from the appearance of strong spin-orbit coupling effects in comparison to heavier elements, such as gold. Pt is just the opposite case: a heavy element with a different electronic configuration, as it has one electron less than gold. The Pt band structure shows the "surface band" associated with the 6s electron above the Fermi level (unoccupied orbital) in the complete $\Gamma \rightarrow \mathrm{M}(\pi / \mathrm{a}, 0,0)$ path. In this way, the sp-band inversion does not cross the Fermi energy, preventing the rich spin texture observed in gold to appear. ${ }^{43}$
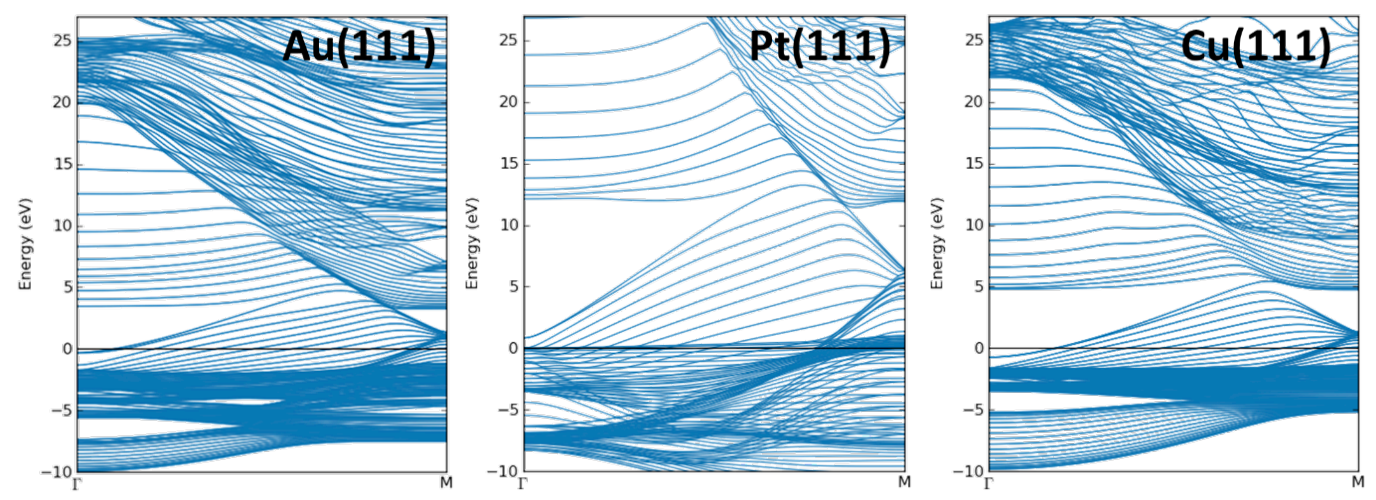

Figure 8. Band diagram along the $\Gamma \rightarrow M$ path for the 18 layer $\mathrm{Au}(111)$ (left) $\mathrm{Pt}(111)$ (centre) and $\mathrm{Cu}(111)$ (right). (All surfaces are 1x1). The Fermi level is indicated by a black line.

\section{CONCLUSIONS}

The present work provides the main ingredients to achieve large magnetoresistance at room temperature in a single-molecule device by exploiting metal complexes. The magnetic nature of the molecule is not the unique requirement to observe the magnetoresistance effect and, in fact, the specific electronic configuration plays the key role. In addition to the top ferromagnetic Ni electrode in the junction, the $\mathrm{Au} /$ molecule spinterface is an essential ingredient in the manifestation 
of the observed single-molecule magnetoresistance, as deduced from the study on the different metal surfaces, namely $\mathrm{Au}, \mathrm{Cu}$ and Pt. The interplay of the surface potential and spin-orbit coupling at the metal surface is of uttermost importance to create the magnetoresistance. The studied spin-dependent transport on single-molecule devices with the metal complex series $\left[\mathrm{M}(\text { tzpy })_{2}(\mathrm{NCSe})_{2}\right](\mathrm{M}=\mathrm{Mn}, \mathrm{Co}$ and $\mathrm{Ni})$ shows that the $\mathrm{Co}^{\mathrm{II}}$ system is the only one presenting magnetoresistance effects in accord with the DFT+NEGF calculations that evidence $\mathrm{Au} / \mathrm{Co}-$ complex hybridized spin-polarized orbitals energetically close to the metal Fermi levels. Overall, these results demonstrate the crucial role of the spinterface ${ }^{42,47}$ defined by the molecule (its electronic structure as spin filter) and the gold surface (spin texture triggered by spin-orbit effects) to provide a rational design of single-molecule Spintronics.

\section{EXPERIMENTAL METHODS}

Sample preparation. An Au (111) single crystal substrate (10 mm x $1 \mathrm{~mm})$ of $99.9999 \%$ purity and orientation accuracy $<0.1$ degrees (Mateck, Germany) was surface-functionalized with the $\left[\mathrm{M}(\text { tzpy })_{2}(\mathrm{NCSe})_{2}\right]$ complexes $(\mathrm{M}=\mathrm{Mn}, \mathrm{Co}$ and $\mathrm{Ni})$ (basic information on the synthesis and characterization can be found in the Supporting Information section 1) by immersion in a $\mu \mathrm{M}$ ethanolic solution of the target compound for periods longer than $6 \mathrm{~h}$. Pt (111) and $\mathrm{Cu}(111)$ single crystal substrates $(10 \mathrm{~mm} \times 1 \mathrm{~mm})$ both of $99.999 \%$ purity and orientation accuracy $<0.1$ degrees (Mateck, Germany) were surface-functionalized only with the $\left[\mathrm{Co}(\operatorname{tzpy})_{2}(\mathrm{NCS})_{2}\right]$ complex by immersion in a $\mu \mathrm{M}$ ethanolic solution of the target compound for periods longer than $6 \mathrm{~h}$. (see SI section 1 for a more detailed preparation). All glassware and PTFE-STM cells were cleaned with piranha solution (3:1 $\mathrm{H}_{2} \mathrm{SO}_{4} / \mathrm{H}_{2} \mathrm{O}_{2}$ by volume) before usage, followed by rinsing with $18 \mathrm{M} \Omega$ $\mathrm{cm}^{-1}$ Milli-Q water (Millipore).

Conductance measurements. An STM-BJ method was employed to build and characterize charge transport through single-molecule wires built with the different metal complexes studied in this 
work $\left(\mathrm{Mn}^{\mathrm{II}}, \mathrm{Ni}^{\mathrm{II}}\right.$ and $\left.\mathrm{Co}^{\mathrm{II}}\right)$. The two biased electrodes of the molecular junction, a $\mathrm{Au}(111)$ single crystal (99.9999\% Mateck, Germany) and a mechanically cut polycrystalline Ni wire (99.99\%, Godfellow, UK) were used as the support and the STM top electrodes, respectively. In a regular STM-BJ experiment (see manuscript), the Ni tip was repeatedly driven onto the $\mathrm{Au}$ surface while simultaneously monitoring the current flowing between them. Several thousands $(\sim 5000)$ retraction curves were then stored and used to build the conductance histogram of the single-molecule device. Because not every curve displayed plateau features corresponding to the molecular quantum conductance of the single-molecule bridge, we designed an automatic algorithm that identify and select curves containing such single-molecule features. The exact same selection criteria were applied throughout all measured series. To avoid the Ni wire oxidation under ambient conditions, the prepared Ni electrode was magnetically polarized and stored under in anaerobic conditions before use, as we report in previous works. ${ }^{21}$ All experiments were conducted in an organic solvent (mesitylene) with very low oxygen and water solubility.

Technical details of the dynamic Break-Junction experiments. Details about the STM-break junction technique are published elsewhere. ${ }^{30}$ All experiments were carried out with a homemade PTFE-STM cell and a PicoSPM II microscope head controlled using a PicoScan 2500 electronics, all from Agilent (USA). The STM head was mechanically and electronically isolated. Data was acquired using a NI-DAQmx/BNC-2110 National Instruments (LabVIEW data acquisition System, USA) and analyzed with our own LabVIEW code. In a typical break-junction experiment, the STM tip is first brought to a tunneling distance over a flat clean metallic surface area. The STM feedback is then turned off and the tip is driven in and out of contact with the substrate at a speed of 1-2 V/s. This 2-points feedback loop is used to collect thousands of current decays (5000-6000) during the tip pulling cycles. In order to minimize possible mechanical disruption of the tip and sample surfaces, the reached maximum current in the 2-points feedback loop was set to a low value well below saturation, which prevented the STM tip crashing against the substrate electrode. $10-15 \%$ of the collected current decays display steps or plateaus, and are used to 
determine the single molecule conductance using the expression $G=I_{\text {step }} / U_{\text {bias }}$ where $G$ is the conductance, I the current and $\mathrm{U}$ is the potential difference between the tip and substrate electrodes. The current decays are accumulated to form linear conductance histograms. The observed peaks in the conductance histograms correspond to the observed plateaus in the current decays and provide averaged single-molecule conductance values. An automated selection process designed by own LabVIEW code was used to select the decays that showed plateaus from the ones that did not. The user defines the initial selection criteria that are fixed throughout all the experimental series.

Technical details of the static blinking experiments. The blinking captures (tens of curves) were accumulated into $2 \mathrm{D}$ maps during a total time of $6 \mathrm{~h}$ for each experiment. ${ }^{34,48}$ No selection procedures were applied at this time and so all blinking events are used to build the 2D histograms. In order to compare the lifetimes of the blinks in the $2 \mathrm{D}$ maps, all the samples were set into a common time origin and baseline. The final STM tip pulling after the blinking was performed by externally controlling the piezo Z-position (see Supporting Information section 5).

Computational details. Electron transport calculations were carried out with the molecule sandwiched between five gold layers with a 4 x 3 surface unit cell using the ATK code $^{36,37}$ (version 2016.1)with the PBE functional, ${ }^{49}$ a numerical single- $\zeta$ basis set and $11 \mathrm{e}^{-}$pseudopotential for the gold atoms ${ }^{50}$ while double- $\zeta$ basis set with polarization for the other elements. The coherent transport properties were calculated using the Non-Equilibrium Green Function procedure combined with the Density Functionl Theory (DFT) calculations. NEVPT2 calculations with quasidegenerate perturbation theory to include spin-orbit effects ${ }^{51}$ were employed to determine the zerofield splitting parameter D for the Co ${ }^{\mathrm{II}}$ complex using Orca 3.0.3 code $^{52}$ and a def2-TZVPP basis set ${ }^{53}$ with an active space considering the seven $d$ electrons of $\mathrm{Co}^{\mathrm{II}}$ centre and the five $\mathrm{d}$ orbitals. Zero bias transmission curves were calculated using the Artaios 1.9 code,${ }^{54,55}$ while the Fock and Overlap matrices necessary for the transport calculations were obtained from unrestricted DFT calculations (B3LYP functional, ${ }^{56}$ D95V basis for $\mathrm{H}, \mathrm{C}$ and $\mathrm{N}$ and LANL2DZ ECP basis set for 
heavier atoms ${ }^{57-60}$ by means of the Gaussian09 package. ${ }^{61}$ Model systems were constructed from previously optimized molecules, attaching 3 gold atoms to each Se end with a Se-Au distance of $2.5 \AA$ (hollow position). Slab calculations including spin-orbit coupling for the $\mathrm{Au}(111), \mathrm{Cu}(111)$ and $\operatorname{Pt}(111)$ surfaces where performed using the green $\operatorname{code}^{62}$ and its interface to the SIESTA package. ${ }^{38}$ The PBE functional ${ }^{49}$ was employed and all atoms were described by a double- $\zeta$ basis set with polarization. Bulk cell parameters were used to build the slabs, without further geometry relaxation. Cell parameters for $1 \times 1$ surfaces were: $(2.8837,0.0) \AA$ and $(-1.4419,2.4974) \AA$ for $\mathrm{Au}(111),(2.5562,0.0)$ and $(-1.2781,2.2137)$ for $\mathrm{Cu}(111)$ and $(2.7744,0.0)$ and $(-1.3872,2.4027)$ for $\operatorname{Pt}(111)$.

\section{ACKNOWLEDGEMENTS}

The research reported here was supported by the Spanish Ministerio de Economía y Competitividad (MINECO) (grants CTQ2015-64579-C3-1-P, CTQ2015-64579-C3-3-P, CTQ2013-46275-P and CTQ2016-78341-P, and Unidad de Excelencia María de Maeztu» MDM-2015-0538) and Generalitat Valenciana (GVPROMETEO2012-049). E.R. thanks Generalitat de Catalunya for an ICREA Academia award. F. J. V.-M. thanks the Spanish (MINECO) for graduate FPI fellowship. I. D.-P. thanks the Ramon y Cajal program (MINECO) for financial support. D.A. thanks CONICYT + PAI "Concurso nacional de apoyo al retorno de investigadores/as desde el extranjero, convocatoria 2014 82140014" for financial support. D.A. thanks Centers of Excellence with Basal/CONICYT financing, grant FB0807, CEDENNA. Powered@NLHPC: This research was partially supported by the supercomputing infrastructure of the NLHPC (ECM-02). The authors acknowledge the general facilities of the University of Barcelona (CCiT-UB) and the computer resources, technical expertise and assistance provided by the Barcelona Supercomputing Centre.

\section{ASSOCIATED CONTENT}

Supporting Information 
Supplementary Information contains chemical compound information and sample preparation information. The Supporting Information is available free of charge on the ACS Publications website.

\section{AUTHOR INFORMATION}

\section{Corresponding Authors}

eliseo.ruiz@qi.ub.es

ismadiezperez@gmail.com

\section{Notes}

The authors declare no competing financial interest.

\section{REFERENCES}

1. Sinova, J.; Valenzuela, S. O.; Wunderlich, J.; Back, C. H.; Jungwirth, T., Rev. Mod. Phys. 2015, 87, 1213-1260.

2. Hirohata, A.; Takanashi, K., J. Phys. D - Appl. Phys. 2014, 47, 193001.

3. Fusil, S.; Garcia, V.; Barthelemy, A.; Bibes, M., Magnetoelectric Devices for Spintronics. In Annual Review of Materials Research, Vol 44, Clarke, D. R., Ed. Annual Reviews: Palo Alto, CA 2014; Vol. 44, pp 91-116.

4. Sanvito, S., Chem. Soc. Rev. 2011, 40, 3336-3355.

5. Bogani, L.; Wernsdorfer, W., Nat. Mat. 2008, 7, 179-186.

6. Mannini, M.; Pineider, F.; Sainctavit, P.; Danieli, C.; Otero, E.; Sciancalepore, C.; Talarico, A. M.; Arrio, M.-A.; Cornia, A.; Gatteschi, D.; Sessoli, R., Nat. Mat. 2009, 8, 194-197.

7. Urdampilleta, M.; Klyatskaya, S.; Cleuziou, J. P.; Ruben, M.; Wernsdorfer, W., Nat. Mat. 2011, 10, 502-506.

8. Vincent, R.; Klyatskaya, S.; Ruben, M.; Wernsdorfer, W.; Balestro, F., Nature 2012, 488, 357360.

9. Ganzhorn, M.; Klyatskaya, S.; Ruben, M.; Wernsdorfer, W., Nat. Nanotech. 2013, 8, 165-169.

10. Bagrets, A.; Schmaus, S.; Jaafar, A.; Kramczynski, D.; Yamada, T. K.; Alouani, M.; Wulfhekel, W.; Evers, F., Nano Lett. 2012, 12, 5131-5136.

11. Schmaus, S.; Bagrets, A.; Nahas, Y.; Yamada, T. K.; Bork, A.; Bowen, M.; Beaurepair, E.; Evers, F.; Wulfhekel, W., Nat. Nanotech. 2011, 6, 185-189.

12. Xie, Z.; Markus, T. Z.; Cohen, S. R.; Vager, Z.; Gutierrez, R.; Naaman, R., Nano Lett. 2011, $11,4652-4655$.

13. Naaman, R.; Waldeck, D. H., Spintronics and Chirality: Spin Selectivity in Electron Transport Through Chiral Molecules. In Annual Review of Physical Chemistry, Vol 66, Johnson, M. A.; Martinez, T. J., Eds. Annual Reviews: Palo Alto, CA, 2015; Vol. 66, pp 263-281.

14. Yeganeh, S.; Ratner, M. A.; Medina, E.; Mujica, V., J. Chem. Phys. 2009, 131, 014707.

15. Göhler, B.; Hamelbeck, V.; Markus, T. Z.; Kettner, M.; Hanne, G. F.; Vager, Z.; Naaman, R.; Zacharias, H., Science 2011, 331, 894-897.

16. Carmeli, I.; Leitus, G.; Naaman, R.; Reich, S.; Vager, Z., J. Chem. Phys. 2003, 118, 1037210375.

17. Vager, Z.; Naaman, R., Chem. Phys. 2002, 281, 305-309.

18. Michaeli, K.; Kantor-Uriel, N.; Naaman, R.; Waldeck, D. H., Chem. Soc. Rev. 2016, 45, 64786487. 
19. Mondal, P. C.; Fontanesi, C.; Waldeck, D. H.; Naaman, R., Acc. Chem. Res. 2016, 49, 25602568.

20. Aragonès, A. C.; Medina, E.; Ferrer-Huerta, M.; Gimeno, N.; Teixidó, M.; Palma, J. L.; Tao, N.; Ugalde, J. M.; Giralt, E.; Díez-Pérez, I.; Mujica, V. Small 2017, 13, 1602519.

21. Aragonès, A. C.; Aravena, D.; Cerdá, J. I.; Acís-Castillo, Z.; Li, H.; Real, J. A.; Sanz, F.; Hihath, J.; Ruiz, E.; Díez-Pérez, I., Nano Lett. 2016, 16, 218-226.

22. Aravena, D.; Ruiz, E., J. Am. Chem. Soc. 2011, 134, 777-779.

23. Baadji, N.; Sanvito, S., Phys. Rev. Lett. 2012, 108, 217201.

24. Bychkov, Y. A.; Rashba, E. I., J. Phys. C-Sol. St. Phys. 1984, 17, 6039-6045.

25. Koga, T.; Nitta, J.; Takayanagi, H., Phys. Rev. Lett. 2002, 88, 126201.

26. Ting, D. Z. Y.; Cartoixa, X., Appl. Phys. Lett. 2002, 81, 4198-4200.

27. Bercioux, D.; Lucignano, P., Rep. Prog. Phys. 2015, 78, 106001.

28. Xu, B.; Tao, N. J., Science 2003, 301, 1221-1223.

29. Hybertsen, M. S.; Venkataraman, L., Acc. Chem. Res. 2016, 49, 452-460.

30. Xia, J. L.; Diez-Perez, I.; Tao, N. J., Nano Lett. 2008, 8, 1960-1964.

31. Chen, F.; Li, X.; Hihath, J.; Huang, Z.; Tao, N., J. Am. Chem. Soc. 2006, 128, 15874-15881.

32. Osorio, H. M.; Catarelli, S.; Cea, P.; Gluyas, J. B. G.; Hartl, F.; Higgins, S. J.; Leary, E.; Low, P. J.; Martín, S.; Nichols, R. J.; Tory, J.; Ulstrup, J.; Vezzoli, A.; Milan, D. C.; Zeng, Q., J. Am. Chem. Soc. 2015, 137, 14319-14328.

33. Vardimon, R.; Klionsky, M.; Tal, O., Nano Lett. 2015, 15, 3894-3898.

34. Aragonès, A. C.; Haworth, N. L.; Ciampi, S.; Bloomfield, N. J.; Wallace, G. G.; Coote, M. L.; Diez-Perez, I.; Darwish, N., Nature 2016, 531, 88-91.

35. Haiss, W.; Wang, C.; Grace, I.; Batsanov, A. S.; Schiffrin, D. J.; Higgins, S. J.; Bryce, M. R.; Lambert, C. J.; Nichols, R. J., Nat. Mater. 2006, 5, 995-1002.

36. QuantumWise A/S, Atomistix ToolKit version 2014.2. 2014, Copenhagen, Denmark.

37. Brandbyge, M.; Mozos, J. L.; Ordejon, P.; Taylor, J.; Stokbro, K., Phys. Review B 2002, 65, 165401.

38. Soler, J. M.; Artacho, E.; Gale, J. D.; Garcia, A.; Junquera, J.; Ordejon, P.; Sanchez-Portal, D., J. Phys.-Cond. Matt. 2002, 14, 2745-2779.

39. Johnson, M., Handbook of Spin Transport and Magnetism. Chapman and Hall/CRC: 2011.

40. Gueddida, S.; Gruber, M.; Miyamachi, T.; Beaurepaire, E.; Wulfhekel, W.; Alouani, M., J. Phys. Chem. Lett. 2016, 900-904.

41. Galbiati, M.; Tatay, S.; Barraud, C.; Dediu, A. V.; Petroff, F.; Mattana, R.; Seneor, P., MRS Bull. 2014, 39, 602-607.

42. Sanvito, S., Nat. Phys. 2010, 6, 562-564.

43. Yan, B.; Stadtmuller, B.; Haag, N.; Jakobs, S.; Seidel, J.; Jungkenn, D.; Mathias, S.; Cinchetti, M.; Aeschlimann, M.; Felser, C., Nat. Commun. 2015, 6, 10167.

44. Gomez-Coca, S.; Cremades, E.; Aliaga-Alcalde, N.; Ruiz, E., J. Am. Chem. Soc. 2013, 135, 7010-7018.

45. Gomez-Coca, S.; Aravena, D.; Morales, R.; Ruiz, E., Coord. Chem. Rev. 2015, 289, 379-392.

46. Gomez-Coca, S.; Urtizberea, A.; Cremades, E.; Alonso, P. J.; Camon, A.; Ruiz, E.; Luis, F., Nat. Commun. 2014, 5, 4300.

47. Barraud, C.; Seneor, P.; Mattana, R.; Fusil, S.; Bouzehouane, K.; Deranlot, C.; Graziosi, P.; Hueso, L.; Bergenti, I.; Dediu, V.; Petroff, F.; Fert, A., Nat. Phys. 2010, 6, 615-620.

48. Haiss, W.; Nichols, R. J.; van Zalinge, H.; Higgins, S. J.; Bethell, D.; Schiffrin, D. J., Phys Chem Chem Phys 2004, 6, 4330-4337.

49. Perdew, J. P.; Burke, K.; Ernzerhof, M., Phys. Rev. Lett. 1996, 77, 3865-3868.

50. Toher, C.; Sanvito, S., Phys. Rev. B 2008, 77, 155402.

51. Atanasov, M.; Aravena, D.; Suturina, E.; Bill, E.; Maganas, D.; Neese, F., Coord. Chem. Rev. 2015, 289, 177-214.

52. Neese, F., Wiley Interdiscip. Rev. Comput. Mol. Sci. 2012, 2, 73-78. 
53. Weigend, F.; Ahlrichs, R., Phys Chem Chem Phys 2005, 7, 3297-3305.

54. Herrmann, C.; Groß, L.; Steenbock, T.; Solomon, G. C. Artaios - a code for postprocessing electronic structure calculations, $1.9 ; 2015$.

55. Herrmann, C.; Solomon, G. C.; Ratner, M. A., J. Phys. Chem. C 2010, 114, 20813-20820.

56. Becke, A. D., J. Chem. Phys. 1993, 98, 5648-5652.

57. Dunning, T. H. J.; Hay, P. J., Gaussian basis sets for molecular calculations. In Modern Theoretical Chemistry, Schaefer III, H. F., Ed. Plenum: New York, 1977; pp 1-28.

58. Hay, P. J.; Wadt, W. R., J. Chem. Phys. 1985, 82, 270-283.

59. Hay, P. J.; Wadt, W. R., J. Chem. Phys. 1985, 82, 299-310.

60. Wadt, W. R.; Hay, P. J., J. Chem. Phys. 1985, 82, 284-298.

61. Frisch, M. J.; Trucks, G. W.; Schlegel, H. B.; Scuseria, G. E.; Robb, M. A.; Cheeseman, J. R.; Scalmani, G.; Barone, V.; Mennucci, B.; Petersson, G. A.; Nakatsuji, H.; Caricato, M.; Li, X.; Hratchian, H. P.; Izmaylov, A. F.; Bloino, J.; Zheng, G.; Sonnenberg, J. L.; Hada, M.; Ehara, M.; Toyota, K.; Fukuda, R.; Hasegawa, J.; Ishida, M.; Nakajima, T.; Honda, Y.; Kitao, O.; Nakai, H.; Vreven, T.; Montgomery, J., J. A.; Peralta, J. E.; Ogliaro, F.; Bearpark, M.; Heyd, J. J.; Brothers, E.; Kudin, K. N.; Staroverov, V. N.; Kobayashi, R.; Normand, J.; Raghavachari, K.; Rendell, A.; Burant, J. C.; Iyengar, S. S.; Tomasi, J.; Cossi, M.; Rega, N.; Millam, N. J.; Klene, M.; Knox, J. E.; Cross, J. B.; Bakken, V.; Adamo, C.; Jaramillo, J.; Gomperts, R.; Stratmann, R. E.; Yazyev, O.; Austin, A. J.; Cammi, R.; Pomelli, C.; Ochterski, J. W.; Martin, R. L.; Morokuma, K.; Zakrzewski, V. G.; Voth, G. A.; Salvador, P.; Dannenberg, J. J.; Dapprich, S.; Daniels, A. D.; Farkas, Ö.; Foresman, J. B.; Ortiz, J. V.; Cioslowski, J.; Fox, D. J. Gaussian 09 (Revision D.01), Wallingford, CT, 2009.

62. Cerda, J., Van Hove, M. A., Sautet, P., Salmeron, M., Phys. Rev. B 1997, 56, 15885-15899. 